\title{
Subtype frequencies, demographic features, and remission rates in juvenile idiopathic arthritis - 265 cases from a Turkish center
}

\author{
Mustafa Çakan ${ }^{1}$, Nuray Aktay-Ayaz ${ }^{1}$, Gonca Keskindemirci², Dilbade Yıldız Ekinci ${ }^{3}$, \\ Şerife Gül Karadağ ${ }^{1}$ \\ Clinics of ${ }^{1}$ Pediatric Rheumatology, ${ }^{2}$ Pediatrics, and ${ }^{3}$ Ophthalmology, Kanuni Sultan Süleyman Research and Training \\ Hospital, Istanbul, Turkey. E-mail: mustafacakan@hotmail.com \\ Received: 22th April 2017, Revised: 8th June 2017, Accepted: 30th June 2017
}

SUMMARY: Çakan M, Aktay-Ayaz N, Keskindemirci G, Ekinci DY, Karadağ ŞG. Subtype frequencies, demographic features, and remission rates in juvenile idiopathic arthritis - 265 cases from a Turkish center. Turk J Pediatr 2017; 59: 548-554.

Juvenile idiopathic arthritis (JIA) is the most common cause of chronic arthritis in children. It is a group of heterogeneous disorders that have chronic arthritis as a common feature. It has a worldwide distribution and many studies have shown that subtype frequencies in JIA seem to be showing geographical distribution. The aim of this study was to define subtype frequencies, demographic features, and the rates of macrophage activation syndrome, uveitis and remission in Turkish JIA patients. The files of all JIA patients (378 cases) that were being followed in Pediatric Rheumatology Clinic of our institution, between May 2010 and February 2016 were reviewed. Two hundred and sixty-five patients were included into the study. Gender, JIA subtype, age at diagnosis, age at the initial symptoms, JIA medications, uveitis presence, JIA status at the time of enrollment were recorded from the files. There were 87 enthesitis related arthritis, 87 oligoarthritis (81 persistent, 6 extended), 36 rheumatoid factor (RF) negative polyarthritis, 35 systemic arthritis, 10 RF-positive polyarthritis, 5 psoriatic arthritis and 5 undifferentiated arthritis cases. Mean age at diagnosis was $9.9 \pm 4.9$ years and male/female ratio was 1.05 . Uveitis was found in $4.5 \%$ of the cases. Biologics were used in $26 \%$ of the patients. At the time of enrollment, $69 \%$ of the patients were under remission while $31 \%$ of them were active. Systemic arthritis and persistent oligoarthritis cases were the groups that most commonly achieved remission, while patients with polyarticular involvement, namely RF positive polyarthritis, RF negative polyarthritis and extended oligoarthritis patients were the groups with high number of active patients. In conclusion, JIA is a heterogeneous group of disorder, and differences in subtype frequencies from country to country make it even more heterogeneous disease. Patients with polyarticular involvement may need early and aggressive treatment to control the disease activity.

Key words: demographics, juvenile idiopathic arthritis, remission, uveitis.

Juvenile idiopathic arthritis (JIA) is the most common cause of chronic arthritis in children and described as arthritis of unknown etiology that begins before the $16^{\text {th }}$ birthday and persists for at least 6 weeks. It is a diagnosis of exclusion, so other known conditions for arthritis must be excluded.1,2 It is not a homogenous disease and has several subtypes. Classification systems were developed and the last one, ILAR (International League of Associations for Rheumatology) classification system that was updated in 2001, divides JIA into 7 subtypes; systemic arthritis, oligoarthritis (persistent and extended), polyarthritis rheumatoid factor (RF) positive, polyarthritis RF negative, psoriatic arthritis, enthesitis related arthritis and undifferentiated arthritis. ${ }^{2}$ There are inclusion and exclusion 
criteria for each group and if a patient fits no category or more than one category then defined as undifferentiated arthritis. ${ }^{2}$ JIA has a worldwide distribution and has an annual incidence between 0.8 and 22.6 per 100,000 children. ${ }^{3}$ The etiology and pathogenesis still remain unclear. It seems to be multifactorial and differ from one subtype to another. ${ }^{1,3}$ Treatment of JIA is multifaceted and includes pharmacotherapy, optimal nutrition, patient and parent education, physical and occupational therapy. ${ }^{1,3,4}$ The introduction of biologics has offered an opportunity to decrease the rates of chronic joint damage, disability and aim for complete disease remission. ${ }^{3}$ Although JIA still cannot be cured, disease control is an achievable goal for many patients, mostly related to JIA subtype. ${ }^{1,3}$

The main objective of this study was to take a small snapshot picture of Turkish children with JIA and compare them with the reports from the literature regarding subtype frequencies, demographic features, and the rates of macrophage activation syndrome, uveitis and remission.

\section{Material and Methods}

The files of all JIA patients (378 cases) that were being followed in our center between May 2010 and February 2016 were reviewed. To be included into the study; the onset of the symptoms related to JIA had to be started before the age of 16 , had to be completed at least initial 6 months of follow-up, had to be coming regularly to the control visits (generally every 3 months), and had to be adherent with the medications and physical therapy. Patients that were diagnosed in the last 6 months or that were dropped off the follow-up or that were not adherent to treatment were excluded (113 patients) and finally 265 cases were included into the study. Gender, JIA subtype, age at diagnosis, age at the initial symptoms, JIA medications, uveitis presence, JIA status at the time of enrollment were recorded from the files. ILAR classification criteria system was used to define JIA subtypes. ${ }^{2}$ All patients were classified at the time of diagnosis of JIA and reclassified after completion of initial 6 months. To define JIA status at the time of enrollment, clinical inactive disease criteria system that was defined by Wallace et al. ${ }^{5}$ was used. Clinical remission with medication was defined as inactive disease for a minimum of 6 consecutive months while the patient is taking medication, and clinical remission without medication was defined as inactive disease for a minimum of 12 consecutive months while the patient is off all anti-arthritis and anti-uveitis medications. To comply with ILAR criteria, all patients were tested for RF and antinuclear antibody (ANA) at the time of diagnosis. RF positivity was verified by a second analysis at least 3 months later. HLA-B27 studies were done in all patients with enthesitis related arthritis phenotype and male patients over the 6 years of age. ANA analysis was done by indirect immunofluorescence assay and titers $\geq 1: 100$ were classified as positive. The study was approved by the local Institutional Review Board (ethics approval number: KAEK/2015.14.11). Informed consent was received from the participants.

Clinical and demographic characteristics were summarized by mean and standard deviation for continuous variables and count and percent for categorical variables. Statistical analyses were performed using the SPSS software package for Windows (version 22.0; SPSS, Chicago, IL, USA).

\section{Results}

There were 265 JIA patients in the cohort. Mean follow-up time was $24.9 \pm 15.5$ months (6 - 68 months). There were 87 enthesitis related arthritis, 87 oligoarthritis (81 persistent, 6 extended), 36 RF-negative polyarthritis, 35 systemic arthritis, 10 RF-positive polyarthritis, 5 psoriatic arthritis and 5 undifferentiated arthritis (Table I). By the completion of initial 6 months of the diagnosis, definitive subtype classification was done and 9 patients showed subtype change. Six oligoarthritis patients that had provisional classification of persistent oligoarthritis turned into extended oligoarthritis. Two cases of persistent oligoarthritis and one case of RF-negative polyarthritis changed into enthesitis related arthritis classification because of the sacroiliac and/or enthesis involvement during the follow-up period. Two persistent oligoarthritis patients had RF positivity and 3 enthesitis related arthritis cases had a history of psoriasis in the first degree relatives and together they formed the 5 cases of the undifferentiated arthritis group. 
Table I. Subtype Frequencies, and Demographic Features of the Patients.

\begin{tabular}{lcccc}
\hline JIA subtype & $\begin{array}{c}\text { Frequency } \\
\mathrm{N}(\%)\end{array}$ & $\begin{array}{c}\text { Gender (male/female) } \\
\mathrm{N} / \mathrm{N}(\% / \%)\end{array}$ & $\begin{array}{c}\text { Age at diagnosis } \\
\text { (years } \pm \text { SD) }\end{array}$ & $\begin{array}{c}\text { Diagnosis delay } \\
\text { (months } \pm \text { SD })\end{array}$ \\
\hline Extended oligoarthritis & $6(2.3)$ & $3 / 3(50.0 / 50.0)$ & $5.9 \pm 4.4$ & $5.0 \pm 3.7$ \\
Persistent oligoarthritis & $81(30.6)$ & $27 / 54(33.3 / 66.7)$ & $7.6 \pm 4.9$ & $5.3 \pm 5.4$ \\
RF-positive polyarthritis & $10(3.8)$ & $4 / 6(40.0 / 60.0)$ & $12.1 \pm 4.5$ & $11.5 \pm 6.5$ \\
RF-negative polyarthritis & $36(13.5)$ & $13 / 23(36.1 / 63.9)$ & $9.5 \pm 5.3$ & $9.6 \pm 9.9$ \\
Enthesitis related arthritis & $87(32.9)$ & $68 / 19(78.1 / 21.9)$ & $13.2 \pm 2.9$ & $8.7 \pm 6.3$ \\
Psoriatic arthritis & $5(1.9)$ & $2 / 3(40.0 / 60.0)$ & $6.6 \pm 3.8$ & $5.2 \pm 4.0$ \\
Systemic arthritis & $35(13.2)$ & $15 / 20(42.8 / 57.2)$ & $8.1 \pm 4.4$ & $1.2 \pm 1.5$ \\
Undifferentiated arthritis & $5(1.9)$ & $4 / 1(80.0 / 20.0)$ & $10.8 \pm 4.6$ & $8.0 \pm 6.9$ \\
\hline Total & 265 & $136 / 129(51.3 / 48.7)$ & $9.9 \pm 4.9$ & $6.7 \pm 6.7$ \\
\hline
\end{tabular}

RF: rheumatoid factor

Male and female ratios were very close to each other in the total cohort (136 male, 129 female; male/female ratio: 1.05/1). While the majority of the enthesitis related arthritis cases and undifferentiated arthritis cases were male, all other groups had a female predominance (Table I). Mean age at diagnosis was $9.9 \pm 4.9$ years (0.8 - 18.0 years) (Table I). Extended oligoarthritis patients were the youngest ones and enthesitis related arthritis patients were the oldest ones at the time of diagnosis. The mean time elapsed from the initiation of the first JIA-related symptom to the diagnosis was $6.7 \pm 6.7(0.5-48.0)$ months. Systemic arthritis patients were the earliest diagnosed group, but RF-positive polyarthritis patients had the longest duration of symptoms until diagnosis (Table I).

ANA positivity was observed in $27.2 \%$ of the cohort. Psoriatic arthritis (60\%), oligoarthritis (44.8\%), and RF-negative polyarthritis (41.6\%) were the groups with a high rate of ANA positivity. HLA-B27 was studied in 169 patients (in all patients with enthesitis related arthritis phenotype and male patients over the 6 years of age) and $26 \%$ of them were positive. There was a significant male predominance, and $82 \%$ of all HLA B-27 positive cases were males. HLA-B27 positivity was found in $45 \%$ of enthesitis related arthritis patients.

Uveitis was found in 12 patients (4.5\%). The majority of the uveitis cases were young girls with persistent oligoarthritis (5 cases) and older boys with enthesitis related arthritis (4 cases). Five of the uveitis cases were ANA positive and 7 of them were ANA negative. All of the cases had anterior uveitis and 10 of them had a single attack. Only one case was initially diagnosed as having uveitis and later developed JIA (enthesitis related arthritis) clinic, and the rest of the uveitis cases were detected during follow-up. At the time of study two cases were classified under the group 'active JIA' because of the ongoing uveitis although all articular and laboratory findings were normal.

Biologics were used in 69 cases (26\%). Extended oligoarthritis (66\%), RF-positive polyarthritis (60\%), psoriatic arthritis (60\%) and RF-negative polyarthritis $(47.2 \%)$ were the groups that most commonly needed a biologic medicine. Only $20 \%$ and $9.8 \%$ of the systemic arthritis and persistent oligoarthritis patients needed biological agent, respectively. Nine patients needed second biological agent: 3 patients were RF-negative polyarthritis and 4 patients were systemic arthritis cases that had a chronic polyarticular course. Four cases needed third or fourth biologic and 3 of them were systemic arthritis cases with a chronic polyarticular course. The mean duration between the first biological agent and the first disease-modifying anti-rheumatic drug was 17.3 \pm 15.3 months (1-60 months).

At the time of enrollment, 183 cases $(69 \%)$ were under remission while 82 cases $(31 \%)$ were active (Table II). Systemic arthritis and persistent oligoarthritis cases were the groups that most commonly achieved remission, while RF-positive polyarthritis, RF-negative polyarthritis and extended oligoarthritis patients were the groups with most active disease. Seventy-two percent of patients were 
Table II. Remission Rates of the Patients.

\begin{tabular}{lcccc}
\hline JIA subtype & Active & In remission & $\begin{array}{c}\text { Remission with } \\
\text { medication }\end{array}$ & $\begin{array}{c}\text { Remission without } \\
\text { medication }\end{array}$ \\
\hline Extended oligoarthritis & $3(50.0)$ & $3(50.0)$ & $3(100)$ & $0(0.0)$ \\
Persistent oligoarthritis & $18(22.2)$ & $63(77.8)$ & $44(69.8)$ & $19(30.2)$ \\
RF-positive polyarthritis & $8(80.0)$ & $2(20.0)$ & $2(100)$ & $0(0.0)$ \\
RF-negative polyarthritis & $18(50.0)$ & $18(50.0)$ & $17(94.4)$ & $1(5.6)$ \\
Enthesitis related arthritis & $27(31.0)$ & $60(69.0)$ & $46(76.6)$ & $14(23.4)$ \\
Psoriatic arthritis & $2(40.0)$ & $3(60.0)$ & $3(100)$ & $0(0.0)$ \\
Systemic arthritis & $4(11.4)$ & $31(88.6)$ & $15(48.3)$ & $16(51.7)$ \\
Undifferentiated arthritis & $2(40.0)$ & $3(60.0)$ & $2(66.7)$ & $1(33.3)$ \\
\hline Total & $82(31.0)$ & $183(69.0)$ & $132(72.1)$ & $51(27.9)$ \\
\hline
\end{tabular}

Data is presented as N (\%). RF: rheumatoid factor

under remission with medication and only $28 \%$ of the patients were under remission without medication. None of the patients with extended oligoarthritis, RF-positive polyarthritis and psoriatic arthritis achieved remission without medication. In 18 patients $(6.8 \%)$ activation of JIA were observed while being followed for a mean of $21.5 \pm 12.4(12-52)$ months under remission without medication.

We did not observe any mortality in the cohort. Thirteen (5\%) of the cases had at least one joint with mild to moderate fixed flexion contracture. Only one patient was using a device to walk. Amyloidosis was not observed in any patient.

\section{Discussion}

Juvenile idiopathic arthritis is an umbrella term that comprises a complex group of disorders with the common feature of arthritis. ${ }^{1,3}$ It has a worldwide distribution and many studies have shown that subtypes of patients with JIA seem to be showing geographical distribution. ${ }^{1}$
It was observed that while oligoarthritis is the most common subtype in Europe and North America, enthesitis related arthritis is the most common subtype in Asia and Middle Eastern countries. $1,6,7,8,9$ The cause and pathogenesis of JIA is still unclear and it is thought that the pathogenesis of each subtype is multifactorial and possibly triggered by environmental stimuli in a genetically susceptible host. Geographical differences in JIA subtype frequencies seem to be supporting this idea and many studies are focusing on the interactions between immune system and microbiome of the gut. ${ }^{1}$ Table III shows the frequency of subtypes from different countries and our study. Equal frequency of oligoarthritis and enthesitis related arthritis in this study may be correlated with the exact geographical location of our country, like a bridge in between Asia and Europe. RF-positive polyarthritis, psoriatic arthritis, and undifferentiated arthritis are the least common subtypes in all ethnic groups and systemic arthritis and RF-negative polyarthritis stand

Table III. Frequencies of Juvenile Idiopathic Arthritis Subtypes in Selected Populations.

\begin{tabular}{lccccc}
\hline JIA subtype & $\begin{array}{c}\text { Our study } \\
\text { (N: 265) }\end{array}$ & $\begin{array}{c}\text { Canada }^{6} \\
(\mathrm{~N}: 366)\end{array}$ & $\begin{array}{c}\text { England }^{7} \\
(\mathrm{~N}: 507)\end{array}$ & $\begin{array}{c}\text { India }^{8} \\
(\mathrm{~N}: 224)\end{array}$ & $\begin{array}{c}\text { Taiwan }^{9} \\
(\mathrm{~N}: 195)\end{array}$ \\
\hline Oligoarthritis & 32.9 & 40.7 & 45.9 & 20.8 & 23.1 \\
RF-positive polyarthritis & 3.8 & 3.7 & 2.3 & 11.9 & 4.6 \\
RF-negative polyarthritis & 13.5 & 20.2 & 13.4 & 17.4 & 11.8 \\
Enthesitis related arthritis & 32.9 & 10.1 & 6.3 & 35.7 & 37.4 \\
Psoriatic arthritis & 1.9 & 6.5 & 6.9 & 1.2 & 1.5 \\
Systemic arthritis & 13.2 & 7.3 & 5.3 & 8.0 & 19 \\
Undifferentiated arthritis & 1.9 & 11.5 & 3.9 & 4.6 & 2.6 \\
\hline
\end{tabular}


with intermediate frequency. ${ }^{6-9}$

All patients in the cohort were classified by the current ILAR system. ${ }^{2}$ ILAR classification system was first described in 1994 and the last update was in 2001.2,10 The main objective of ILAR system was to create more homogenous subtypes in a heterogeneous disease like JIA for clinical and genetic studies. It has strict inclusion and exclusion criteria. After completion of initial 6 months, patients were reclassified and $6(6.8 \%)$ of 87 oligoarthritis patients turned out to be extended oligoarthritis. The ratio of extended oligoarthritis patients in our cohort $(6.8 \%)$ is less than the literature. Kunjir et al. ${ }^{8}$ reported that $20.4 \%$ of oligoarthritis patients were extended oligoarthritis, and this ratio was $28.9 \%$ in the study of Shen et al. ${ }^{9}$ A nationwide study done in Turkish children found that the ratio of extended oligoarthritis was $10 \%$, that is close to our study and less frequent as compared to the literature. ${ }^{11}$ This finding may be attributed to relatively small sample size in our total cohort.

Like many other connective tissue diseases, JIA is reported to be more common in girls. This is true for European, and North American patients where twice as many girls as boys are affected, but reports from Asia and Turkey state that male and female JIA patients seem to be equal or have slightly female predominance. $1,8,9,11,12$ Nearly equal frequency of gender distribution in our cohort may be explained by high frequency of enthesitis related arthritis patients in our country which is much more common in males.

The term juvenile refers to people under the age of 16 , and in some countries adult rheumatologist take care of the children over 16 years of age and in some over 18. ${ }^{2}$ This upper limit for age is one of the debated topics in the definition of JIA. In our country a person aged equal to or less than 18 years must be seen by a pediatrician. So we had 29 cases of JIA diagnosed between the ages of 16 and 18 . We have classified them as JIA because in all of them arthritis symptoms started before the $16^{\text {th }}$ birthday, which was in accordance with definition of JIA by ILAR criteria. ${ }^{2}$ Mean age at diagnosis was 9.9 years in our study and mean time to diagnosis after the first JIA-related symptom was 6.7 months. Time passed from onset to diagnosis is longer than the cohorts of developed countries. In Canada it was found to be 4.5 months and in England 4.6 months. ${ }^{6,7}$ In Turkey, fellowship education program in pediatric rheumatology was installed in 2012. Twenty-eight patients in our cohort were diagnosed 18 months or later from the first symptom of arthritis and many of these cases were coming from Anatolian cities that were located far away from pediatric rheumatology centers. Thus, this lag time could be partly explained by the low number of pediatric rheumatology centers in Turkey. Hopefully this trend will change in the near future as the number of young pediatric rheumatologists is increasing and future studies will be needed to compare these results.

Macrophage activation syndrome (MAS) is the most dreadful complication of systemic arthritis and the leading cause of JIA-related mortality. ${ }^{13}$ In $34 \%$ of systemic arthritis cases we have observed MAS. MAS frequency in systemic arthritis patients is reported to be between 7\%$12.6 \% .14,15$ The frequency of MAS in our cohort is much higher than the literature (34\%), but we are very aware of clinical and laboratory signs of an impending MAS attack, so we closely follow and evaluate every systemic arthritis patient for MAS development. Ravelli et al. ${ }^{16}$ reported that, in addition to the approximately $10 \%$ risk of developing overt MAS as part of systemic arthritis, another $30 \%-40 \%$ of systemic arthritis patients may have occult or subclinical MAS during disease course that can eventually lead to overt MAS. This statement is compatible with the high number of MAS patients in our cohort.

Uveitis is a major concern for all pediatric rheumatologists in JIA patients as we depend on the reports of another doctor, ophthalmologist. There are two patterns of uveitis; one with insidious onset, mostly asymptomatic, bilateral, chronic non-granulomatous anterior uveitis that is more common in young girls with ANA positive oligoarthritis. The other group have sudden onset, symptomatic, mostly unilateral acute anterior uveitis that is most commonly seen in older boys with enthesitis related arthritis. ${ }^{17}$ It was stated that ANA positivity could be found in $70 \%-80 \%$ of oligoarthritis patients and uveitis could develop in up to $45 \%$ of patients. ${ }^{17}$ Our cohort included only $12(4.5 \%)$ uveitis patients. It was shown in previous studies that uveitis is more common 
in developed countries. Uveitis frequency in Netherlands was found to be $17.6 \%$ and $20.1 \%$ in Italy. ${ }^{18,19}$ In our clinic uveitis screening was being done by an ophthalmologist experienced in pediatric uveitis every 3 or 6 months depending on the JIA subtype and ANA status. So missing the uveitis cases could not simply explain low uveitis rate in our cohort but low ANA positivity in the total cohort $(27.2 \%)$ and in oligoarthritis patients $(44.8 \%)$ could be the reason for low number of uveitis.

The aim of treatment in JIA is to suppress the ongoing inflammation as soon as possible to achieve remission and prevent long-term damage. 1,3 At the time of enrollment $69 \%$ of the cases were under remission while $31 \%$ of them were active. Patients with polyarticular involvement namely, RF-positive polyarthritis, RF-negative polyarthritis, and extended oligoarthritis patients which were also the subtypes mostly needed a biologic treatment were the groups with high number of active patients. Although 31 (89\%) systemic arthritis patients achieved remission, the remaining four patients were the ones that we have struggled most in the treatment. All four patients initially showed a polycyclic course and later followed a polyarticular course. The review by Ravelli and Martini ${ }^{20}$ demonstrated that the percentage of patients in clinical remission at the last follow-up ranged from $33 \%$ to $80 \%$ for systemic arthritis; $0 \%$ to $15 \%$ for RF-positive polyarthritis; $23 \%$ to $46 \%$ for RFnegative polyarthritis; $12 \%$ to $35 \%$ for extended oligoarthritis; and $43 \%$ to $73 \%$ for persistent oligoarthritis. They stated that the long-term outcome was best in persistent oligoarthritis and worst in RF-positive polyarthritis. They also stated that the outcome in systemic arthritis is widely variable, perhaps reflecting the considerable heterogeneity of this JIA subtype. Our remission rates seem to be in accordance with the reported ones in the literature.

The drawbacks of our study seem to come from the relatively low number of patients, especially in some subtypes, and short follow-up time.

In conclusion, JIA is a heterogeneous group of disorders that present with chronic arthritis. Differences in subtype frequencies from country to country make it an even more heterogeneous disease. High rates of enthesitis related arthritis in developing countries may reflect the importance of environmental factors, e.g. microbiome of the gut, in the pathogenesis of JIA. ANA seems to be an important risk factor for uveitis development as reflected by low number of ANA positivity and low number of uveitis in our cohort. Patients with polyarticular involvement and systemic arthritis patients that follow a chronic polyarticular course seem to be the most difficult cases in suppressing ongoing inflammation and may need earlier and more aggressive treatment approaches.

\section{REFERENCES}

1. Petty RE, Laxer RM, Lucy R, Wedderburn LR. Juvenile Idiopathic Arthritis. In: Petty RE, Laxer RM, Lindsey CB, Wedderburn LR (eds). Textbook of Pediatric Rheumatology (7th ed). Philadelphia: Elsevier, 2016: 188-204.

2. Petty RE, Southwood TR, Manners P, et al. International League of Associations for Rheumatology classification of juvenile idiopathic arthritis: Second revision, Edmonton, 2001. J Rheumatol 2004; 31: 390-392.

3. Gowdie PJ, Tse SM. Juvenile idiopathic arthritis. Pediatr Clin North Am 2012; 59: 301-327.

4. Laxer RM, Sherry DD, Hashkes PJ. Juvenile Idiopathic Arthritis (JIA). In: Laxer RM, Sherry DD, Hashkes PJ (eds). Pediatric Rheumatology in Clinical Practice (2nd ed). London: Springer, 2016: 31-62.

5. Wallace CA, Huang B, Bandeira M, Ravelli A, Giannini EH. Patterns of clinical remission in select categories of juvenile idiopathic arthritis. Arthritis Rheum 2005; 52: 3554-3562.

6. Oen K, Tucker L, Huber AM, et al. Predictors of early inactive disease in a juvenile idiopathic arthritis cohort: results of a Canadian multicenter, prospective inception cohort study. Arthritis Rheum 2009; 61: 1077-1086.

7. Adib N, Hyrich K, Thornton J, et al. Association between duration of symptoms and severity of disease at first presentation to paediatric rheumatology: Results from the Childhood Arthritis Prospective Study. Rheumatology (Oxford) 2008; 47: 991-995.

8. Kunjir V, Venugopalan A, Chopra A. Profile of Indian patients with juvenile onset chronic inflammatory joint disease using the ILAR classification criteria for JIA: A community-based cohort study. J Rheumatol 2010; 37: 1756-1762.

9. Shen CC, Yeh KW, Ou LS, Yao TC, Chen LC, Huang JL. Clinical features of children with juvenile idiopathic arthritis using the ILAR classification criteria: A community-based cohort study in Taiwan. J Microbiol Immunol Infect 2013; 46: 288-294.

10. Fink CW. Proposal for the development of classification criteria for idiopathic arthritides of childhood. J Rheumatol 1995; 22: 1566-1569.

11. Demirkaya E, Ozen S, Bilginer Y, et al. The distribution of juvenile idiopathic arthritis in the eastern Mediterranean: Results from the registry of the Turkish Paediatric Rheumatology Association. Clin Exp Rheumatol 2011; 29: 111-116. 
12. Unsal E, Oren O, Salar K, et al. The frequency of autoimmune thyroid disorders in juvenile idiopathic arthritis. Turk J Pediatr 2008; 50: 462-465.

13. Boom V, Anton J, Lahdenne P, et al. Evidence-based diagnosis and treatment of macrophage activation syndrome in systemic juvenile idiopathic arthritis. Pediatr Rheumatol Online J 2015; 13: 55.

14. De Benedetti F, Schneider R. Systemic Juvenile Idiopathic Arthritis. In: Petty RE, Laxer RM, Lindsey CB, Wedderburn LR (eds). Textbook of Pediatric Rheumatology (7th ed). Philadelphia: Elsevier, 2016: 205-216.

15. Huang H, Qian X, Yu H, Li J, Zhang Y. Clinical analysis in 202 children with juvenile idiopathic arthritis. Clin Rheumatol 2013; 32: 1021-1027.

16. Ravelli A, Grom AA, Behrens EM, Cron RQ. Macrophage activation syndrome as part of systemic juvenile idiopathic arthritis: diagnosis, genetics, pathophysiology and treatment. Genes Immun 2012; 13: $289-298$
17. Tugal-Tutkun I, Quartier P, Bodaghi B. Disease of the year: Juvenile idiopathic arthritis-associated uveitisclassification and diagnostic approach. Ocul Immunol Inflamm 2014; 22: 56-63.

18. Reininga JK, Los LI, Wulffraat NM, Armbrust W. The evaluation of uveitis in juvenile idiopathic arthritis (JIA) patients: Are current ophthalmologic screening guidelines adequate? Clin Exp Rheumatol 2008; 26: 367-372.

19. Grassi A, Corona F, Casellato A, Carnelli V, Bardare M. Prevalence and outcome of juvenile idiopathic arthritis-associated uveitis and relation to articular disease. J Rheumatol 2007; 34: 1139-1145.

20. Ravelli A, Martini A. Remission in juvenile idiopathic arthritis. Clin Exp Rheumatol 2006; 24: S105-S110. 